\title{
Volume determination for bulk materials in bunkers
}

\author{
S. A. Ahmed ${ }^{1}$, R. Buckingham ${ }^{2}$, P. A. Gremaud ${ }^{3, *}$, C. D. Hauck ${ }^{4}$, C. M. Kuster ${ }^{3}$, \\ M. Prodanovic ${ }^{5}$, T. A. Royal ${ }^{6}$, V. Silantyev ${ }^{7}$ \\ 1 Department of Mathematics and Statistics, Utah State University, Logan, UT 84322-3900, USA. \\ 2 Department of Mathematics, Duke University, Durham, NC 27708-0320, USA. \\ 3 Department of Mathematics and Center for Research in Scientific Computation, North Carolina State \\ University, Raleigh, NC 27695-8205, USA. \\ 4 Department of Mathematics, University of Maryland, College Park, MD 20742-4015, USA. \\ 5 Department of Applied Mathematics and Statistics, SUNY at Stony Brook, Stony Brook, NY 11794-3600, \\ USA. \\ 6 Jenike $\&$ Johanson, inc., One Technology Drive, Westford, MA 01886, USA. \\ 7 Department of Mathematics, Northeastern University, Boston, MA 02115, USA.
}

\section{SUMMARY}

A simple model for the determination of the shape of large granular piles in complicated geometries is discussed. An eikonal formulation of the problem is proposed. Two distinct cases arise. In cylindrical geometries, i.e., if both container and possible obstacles have vertical walls, the problem is equivalent to a two-dimensional traveltime problem with obstacles, while in general geometries, this analogy breaks down. In the first case, classical one-sided discretizations are generalized to handle obstacles without loss in accuracy. In the second case, a fast and efficient numerical method is proposed, implemented and tested. The discrete problems are solved through fast marching.

KEY WORDS: granular; eikonal formulation; ordered upwind methods

\section{INTRODUCTION}

Countless raw materials are stored in granular form in silos and bins of various shapes, from construction materials and ores to food products and pharmaceutical powders. Retrieval is usually achieved by letting the material flow out of the container, by the action of gravity, through outlets at the bottom of the structure. A deceptively simple problem is the determination of the volume of material inside such containers when those have possibly complicated geometries including inserts, internal walls, etc... In practice, one or several gauges measure the height of material at given points. In this paper, a robust, accurate and efficient

* Correspondence to: P.A. Gremaud, Department of Mathematics, North Carolina State University, Raleigh, NC 27695-8205, USA.

Contract/grant sponsor: The third author was partially supported by the National Science Foundation (NSF); contract/grant number: DMS-9818900 and DMS-02044578. 
method for the numerical determination of the geometry of the top free surface, and hence the volume occupied by the material, is proposed. The above problem has three main aspects: i) modeling of granular piles, ii) appropriate mathematical formulation of problems with possibly complicated obstacles, iii) numerical strategy. While addressing all three aspects, the original contributions of this paper are mainly related to ii) and iii).

For future reference, the slope of the pile at a given point on the free surface is defined as the tangent of the angle $\theta$ that the normal vector to the surface makes with the vertical direction. The angle of repose is the largest value of $\theta$ corresponding to a stable configuration. The study of granular piles has a long history, largely due to its connection to soil mechanics in civil and military engineering $[8,14]$. Coulomb [8] was the first to relate the angle of repose of a material to its frictional properties. Since then, a lot of research has been devoted to the physical understanding of sand pile formation and avalanches, see for instance [3, 4, 6, 7, 16]. Various mathematical models have been proposed $[1,2]$ and especially [16] where comparisons are discussed. Under some circumstances, the slope of a growing heap may become time dependent [12]; impact of falling particles at the top and interaction with walls at the bottom may also result in departure from constant slope (tails) $[13,16]$. While addressing some of those points, the above models are generally limited to simple low dimensional geometries.

The present application deals with large piles in quasistationary states. In other words, we are not interested in the dynamics of the pile formation and/or the precise avalanche process. Under those assumptions, the angle of repose for cohesionless material can be shown to be equal to the angle of internal friction $\delta$ [18], which is defined by $\mu=\tan \delta, \mu$ being the coefficient of internal friction.

Modeling issues are treated in Section 2. The numerical method of resolution is described in Section 3 while computational results are presented in Section 4. Finally, conclusions are offered in Section 5.

\section{MODELING}

To simplify, only one point source of material is considered. Several sources can be handled by repeating the approach presented here as many times as there are sources and postprocessing appropriately. A system of Cartesian coordinates $\{x, y, z\}$ is introduced and we assume for now that the source of material is located above the origin $(0,0,0)$. Knowing the height of material $H$ below the source, we aim at finding the shape of the heap of material. More precisely, the material is assumed to occupy the domain

$$
\{(x, y, z) ; h(x, y)<z<u(x, y)\},
$$

where $h$ describes a known support surface (bottom) and $u$ is the unknown height of material. According to the remarks in the Introduction, the angle between the normal vector to the free surface $z=u(x, y)$ and $\langle 0,0,1\rangle$ is assumed to be equal to $\delta$; equivalently

$$
|\nabla u|=\tan \delta .
$$

If the support surface $z=h(x, y)$ satisfies $|\nabla h|<\tan \delta$ (no obstacle), the solution to the problem is trivially a regular cone where the function $u$ in (1), expressed here in polar coordinates $(r, \phi)$ for convenience, is given by

$$
u(r, \phi)=\max \{H-r \tan \delta, h(r \cos \phi, r \sin \phi)\} .
$$




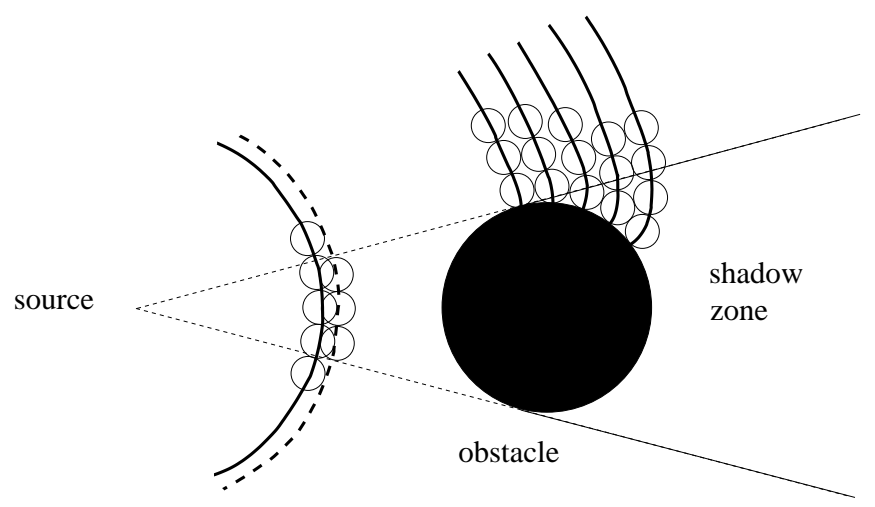

Figure 1. The Huygens principle at play in the two dimensional case.

We now turn to problems with obstacles or, equivalently, the simplifying assumption $|\nabla h|<\tan \delta$ is dropped. It is helpful to distinguish between two cases: cylindrical obstacles which lead to an essentially two dimensional formulation and are standard, and non-cylindrical obstacles which are not.

\section{Cylindrical obstacles}

A cylindrical obstacle is a domain in which no material can enter which is of the form $Q=\{(x, y, z) ;(x, y) \in \Omega, z \geq h(x, y)\}$, where $\Omega$, a two-dimensional domain, is the (constant) horizontal cross-section of $Q$. This case corresponds to standard two dimensional traveltime problems $[19,24]$. The height $u(x, y)$ of the material at a point $P(x, y)$ can be related to the time $T(x, y)$ it would take a signal emited at the source to reach that point. The fixed slope $\tan \delta$ of the heap corresponds to a fixed rate of increase of $T$. Following this analogy, the speed of propagation is then $1 / \tan \delta$. The solution can be constructed according to the classical Huygens principle. The wavefront of a propagating wave emanating from the source conforms, at any instant, to the envelope of spherical wavelets emanating from every point on the wavefront at the prior instant (assuming the wavelets have the same speed as the overall wave). Figure 1 illustrates this principle in the case of a circular right cylindrical obstacle. In the visible part of the domain, i.e., at points that can "see" the source, the exact solution is a cone of half opening angle $\pi / 2-\delta$, hence the level lines are circular. In the shadow zone, those lines are bent as the wavefront turns around the obstacle.

To find the height of material at a point $P(x, y)$, the problem essentially boils down to finding the shortest distance $d(P, O)$ bewteen $P$ and the source $O(0,0)$, i.e.

$$
d(P, O)=\inf \left\{\int_{0}^{1}\left|\frac{d \gamma}{d s}(s)\right| d s, \gamma \in \mathrm{C}^{1}\left([0,1] ; \mathrm{R}^{2}\right), \gamma(s) \in R^{2} \backslash \Omega \quad \forall s \in[0,1], \gamma(0)=O, \gamma(1)=P\right\} .
$$

For some simple cylindrical obstacles, explicit solutions can be found. Figure 2 illustrates the exact shape of the free surface around a circular cylindrical obstacle.

For general cylindrical obstacles, the efficient calculation of $d(\cdot, \cdot)$ is at the heart of the problem and is, by itself, still an area of research in computational geometry, see for instance [15] and the references therein. Rather than attacking this problem directly, we propose an Eikonal formulation. The obstacle is characterized by the fact that the material (or wavefront) 


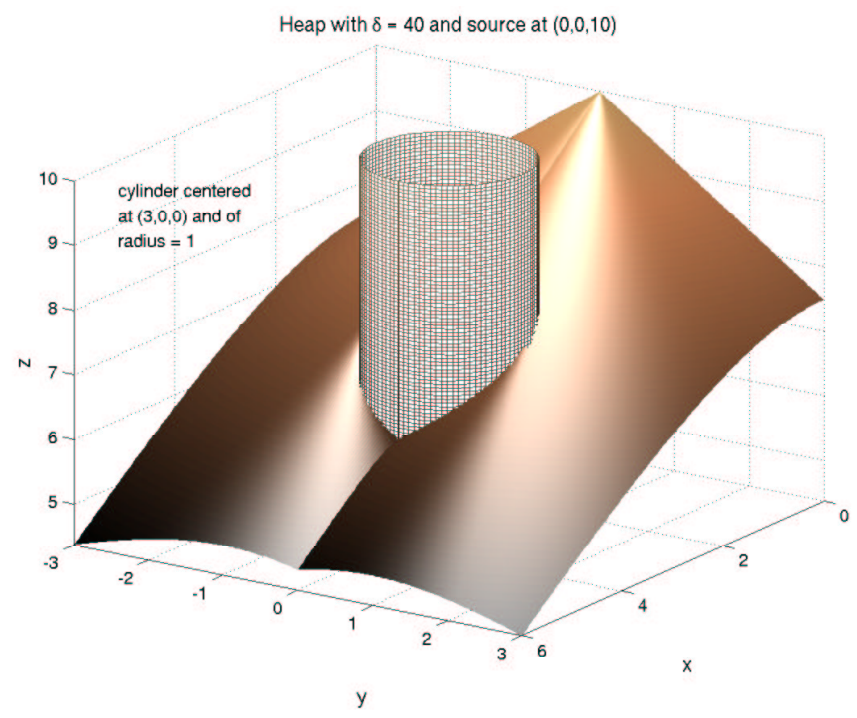

Figure 2. Exact heap around a circular cylindrical obstacle for a material with an angle of repose of $\delta=40^{\circ}$

cannot penetrate it. Equivalently, this corresponds to a vanishing speed of propagation there. If $F$ denotes the slowness, i.e., the inverse of the speed, we set

$$
F(x, y)= \begin{cases}\tan \delta & \text { if }(x, y) \notin \Omega \\ \infty & \text { if }(x, y) \in \Omega\end{cases}
$$

The traveltime $T$ then satisfies

$$
\begin{aligned}
|\nabla T| & =F \quad \text { in } \mathrm{R}^{2} \\
T(0,0) & =0 .
\end{aligned}
$$

We have therefore recast our problem into finding $T$ that solves $(3,4)$ from which the height $u$ is easily obtained through

$$
u(x, y)=\max \{H-T(x, y), h(x, y)\} .
$$

Equation (3) is the Eikonal equation, the simplest form of Hamilton-Jacobi problems. Such a formulation is typical of traveltime problems, for instance related to geophysics [24].

The Huygens principle can be viewed as a selection mechanism that picks among all the (weak) solutions to $(3,4)$ the one that respects causality or, in other words, the fact that information propagates "one way". There is no mechanism in $(3,4)$ that ensures $T$ to go from smaller values to larger values when moving outwards from $O$ ("sawtooth" solutions are possible). The relevant solution is well known to be the viscosity solution $[9,10,19]$.

\section{Non-cylindrical obstacles}

General three dimensional obstacles raise additional problems. First, the analogy with traveltime problems breaks down. In standard three dimensional traveltime problems [20], 


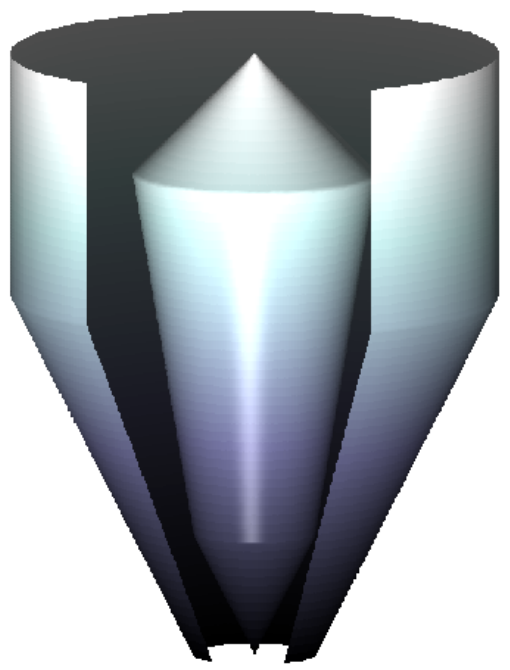

Figure 3. Bin geometry with insert.

level sets of the time of propagation $T$ are surfaces and all spatial dimensions essentially play an identical role. Our problem is clearly different in that level sets for $T$ or the height $u$ are still curves, as in the previous two-dimensional case. The only difference between our two and three dimensional formulations is the role played by the obstacle. Second, as before, one solves $(3,4)$ but now the slowness $F$ itself is a function of the unknown $T$, i.e.

$$
F(x, y, T)= \begin{cases}\tan \delta & \text { if }(x, y, u(x, y)) \notin Q, \text { where } u(x, y)=\max \{H-T(x, y), h(x, y)\} . \\ \infty & \text { otherwise. }\end{cases}
$$

Third, in the general case, the $x, y$-coordinates of the top of the heap will change with increasing values $H$, see for instance Figure 3 .

\section{NUMERICS}

The numerical resolution of the Eikonal equation (3) relies on two fundamental steps. First, the gradient operator is replaced by some appropriate finite difference formula involving values at the nodes of a two-dimensional mesh. Then, the resulting nonlinear system is solved. Fast Marching or ordered upwinding $[11,19,22,23]$ provides an efficient way to perform the last step. The discretization is first described for problems with cylindrical obstacles.

\section{Discretization (cylindrical case)}

The nodes used are defined on a uniform Cartesian mesh away from the obstacle. Near the obstacle, additional nodes corresponding to the intersection of the obstacle's boundary with the mesh lines are added, see Figure 4. The step is necessary in order to avoid severe loss in 


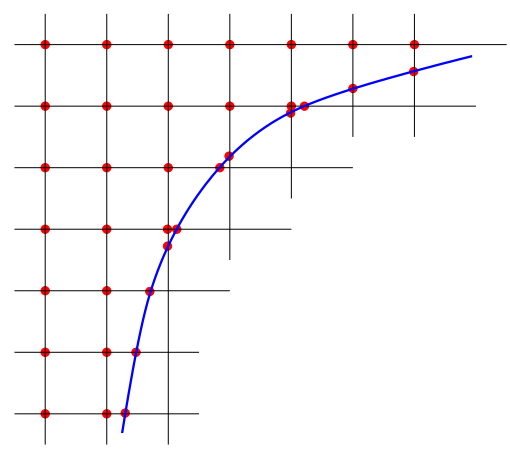

Figure 4. Mesh structure around the obstacle.

accuracy, see below. The method presented below generalizes the one from [21, 22] to problems with obstacles. A different and first order approach is briefly discussed in [19], Section 7.1.

Consider a node $X_{0}$ at which the solution $T$ is to be computed. Assume the values of $T\left(T_{i}\right.$, $i=1,2)$ and its derivatives $\left(\partial_{x} T_{i}, \partial_{y} T_{i}, i=1,2\right)$ are known (or have already been computed) at the neighboring nodes $X_{1}$ and $X_{2}$. We set

$$
N_{i}=\frac{X_{0}-X_{i}}{\left|X_{0}-X_{i}\right|}, \quad i=1,2 \quad \text { and } \quad N=\left[\frac{N_{1}}{N_{2}}\right],
$$

where $N$ is a $2 \times 2$ nonsingular matrix, assuming $X_{0}, X_{1}$ and $X_{2}$ are not lined up. The directional derivatives $N_{1}$ and $N_{2}$ are approximated by

$$
\begin{aligned}
D_{i} T & =\frac{T_{0}-T_{i}}{\left|X_{0}-X_{i}\right|} \quad i=1,2 \quad \text { (1st order formula) } \\
D_{i} T & =2 \frac{T_{0}-T_{i}}{\left|X_{0}-X_{i}\right|}-N_{i} \cdot\left[\partial_{x} T_{i}, \partial_{y} T_{i}\right] \quad i=1,2 \quad \text { (2nd order formula). }
\end{aligned}
$$

Those approximate directional derivatives are linked to the gradient by

$$
D_{i} T=N_{i} \cdot \nabla T+\mathcal{O}\left(\left|X_{0}-X_{i}\right|^{\alpha}\right) \quad \text { or } \quad D T=N \nabla T+\mathcal{O}\left(h^{\alpha}\right),
$$

where $D T=\left[D_{1} T, D_{2} T\right], h=\max \left\{\left|X_{0}-X_{1}\right|,\left|X_{0}-X_{2}\right|\right\}$, and $\alpha=1$ or 2 depending on whether (5) or (6) is respectively used. Solving for $\nabla T$ and plugging into (3), one finds the (quadratic) equation defining the unknown $T_{0}$

$$
D T^{t}\left(N N^{t}\right)^{-1} D T=F\left(X_{0}\right) .
$$

If $\theta$ denotes the angles between $N_{1}$ and $N_{2}$, note that the matrix $N N^{t}$ is symmetric positive definite provided $0<\theta<\pi$; its condition number is $\frac{1+\cos \theta}{1-\cos \theta}$. Therefore, regardless of the choice of stencil (first or second order) (8) has two real solutions out of which we always consider the larger one only, to be consistent with causality. Following [22], we also consider as an upwinding criterion the condition that the characteristic direction should point into the simplex defined by $X_{0}, X_{1}$ and $X_{2}$. This is equivalent to requiring the approximate gradient defined by (7) to have positive components when expressed in terms of the unit vectors $N_{1}$ and $N_{2}$ or, using the above notation

$$
\left(N N^{t}\right)^{-1} D T>0
$$


Therefore, the value $T_{0}$ of $T$ at $X_{0}$ is determined as follows

$$
T_{0}=\left\{\begin{array}{c}
\begin{array}{c}
\text { solution of (8) with } 2 \text { nd order stencil (6) } \\
\text { otherwise }
\end{array} \\
\begin{array}{c}
\text { if (9) is satisfied } \\
\text { solution of (8) with 1st order stencil (5) } \\
\text { otherwise }
\end{array} \\
\begin{array}{c}
\text { if (9) is satisfied } \\
\min _{i=1,2}\left\{T_{i}+\left|X_{0}-X_{i}\right| F\left(X_{0}\right)\right\}
\end{array}
\end{array}\right.
$$

In the first two cases, the values of the partial derivatives of $T$ at $X_{0}$ are updated using $\nabla T=N^{-1} D T$. In the third case, we set $\nabla T=N_{i} F\left(X_{0}\right)$, where $i$ is the index corresponding to the case of lowest value for $T_{0}$.

\section{Discretization (non-cylindrical case)}

In the cylindrical case, the free material surface intersects the obstacle along a space curve $\mathcal{C}$ of a priori unknown geometry, see again Figure 2. The projection of $\mathcal{C}$ in the $x, y$-plane is however obvious: it is part or of all the horizontal cross section of the obstacle. In the non-cylindrical case, the projection of $\mathcal{C}$ itself is unknown. Therefore, the mesh cannot be a priori adapted to fit this projection as done for cylindrical obstacles.

A similar approach can be nevertheless be designed. New nodes lying on the $x, y$-projection of the curve $\mathcal{C}$ can be generated by using local information (value and gradient of the numerical solution) at computed nodes having neighbors inside the obstacle. This approach requires on the fly updating of the mesh's connectivity table. Its implementation will be the object of a future publication. The results presented below for non-cylindrical obstacle were obtained on non-modified meshes.

\section{Fast marching}

Following [11], the nodes are divided into three sets: at the Accepted nodes, the solution is assumed to be known. The Close nodes are the primary neighbors of the Accepted nodes that are not Accepted. The remaining nodes are Far.

As observed in [17], in the presence of a single source point, a singularity of the travel time field is clearly located at the source itself. Unless special care is taken near the source, a loss of accuracy ensues. The problem is fixed here by defining a mesh independent domain around the source and initializing the values at the nodes there to the corresponding values of the exact solution. Initially, the Accepted list is made of the nodes in that domain; the Close list contains their primary neighbors which are not in Accepted. To compute the value at a node in Close, the quadratic equation (8) is solved for all possible Accepted neighbor (1st order formula, (5)) or pair of Accepted neighbors (2nd order formula, (6)). At each node, the smallest of the possible computed value is retained. The Far nodes are initially set to $\infty$.

The solution is then propagated from the neighborhood of the source out in a way consistent with the Huygens principle. After this initialization step, the algorithm proceeds in a standard way as follows

1. the lowest point in Close is removed from that list added to Accepted; its Far neighbors are removed from Far and added to Close;

2. the values of all the Close neighbors of the chosen node are recomputed by solving (8) as explained above; 


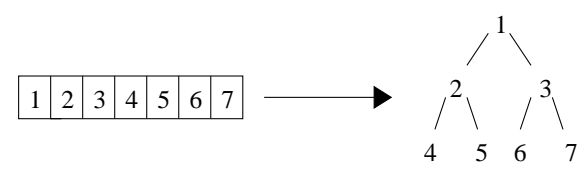

Figure 5. Interpretation of array as a tree structure

\begin{tabular}{|l|l|l|l|l|l|l|l|l|l|l|l|l|}
\hline & \multicolumn{9}{|c|}{ standard stencil } & \multicolumn{9}{c|}{ modified stencil } \\
\hline $\mathrm{N}$ & $L^{1}$ & rate & $L^{2}$ & rate & $L^{\infty}$ & rate & $L^{1}$ & rate & $L^{2}$ & rate & $L^{\infty}$ & rate \\
\hline 25 & $2.91(0)$ & & $9.00(-1)$ & & $6.11(-1)$ & & $1.13(0)$ & & $3.04(-1)$ & & $1.44(-1)$ & \\
50 & $1.71(0)$ & .77 & $5.24(-1)$ & .78 & $3.52(-1)$ & .79 & $7.58(-1)$ & .58 & $2.00(-1)$ & .60 & $9.18(-2)$ & .65 \\
100 & $1.07(0)$ & .68 & $3.27(-1)$ & .68 & $2.23(-1)$ & .66 & $4.41(-1)$ & .78 & $1.13(-1)$ & .82 & $4.92(-2)$ & .88 \\
200 & $6.42(-1)$ & .74 & $2.00(-1)$ & .71 & $1.46(-1)$ & .61 & $2.39(-1)$ & .88 & $6.10(-2)$ & .89 & $2.65(-2)$ & .91 \\
400 & $3.76(-1)$ & .77 & $1.20(-1)$ & .74 & $9.36(-2)$ & .64 & $1.27(-1)$ & .92 & $3.24(-2)$ & .91 & $2.02(-2)$ & .39 \\
800 & $2.21(-1)$ & .77 & $7.24(-2)$ & .73 & $6.07(-2)$ & .62 & $6.38(-2)$ & .99 & $1.64(-2)$ & .99 & $1.54(-2)$ & .39 \\
1600 & $1.31(-1)$ & .76 & $4.40(-2)$ & .72 & $3.82(-2)$ & .67 & $3.07(-2)$ & 1.1 & $7.91(-3)$ & 1.0 & $1.14(-2)$ & .43 \\
3200 & $7.80(-2)$ & .75 & $2.68(-2)$ & .72 & $2.44(-2)$ & .65 & $1.49(-2)$ & 1.0 & $3.88(-3)$ & 1.0 & $6.77(-3)$ & .75 \\
\hline
\end{tabular}

Table I. Convergence study for formally first order methods in the presence of a cylindrical obstacle

\section{3. go back to 1 .}

The list operations are handled by the heap sort algorithm [5, 25], as suggested for instance in [19], which is the key to the speed of the fast marching method. The idea behind the heap sort is to view a 1-D array as a binary tree (Figure 5). This is done by defining the following for node $k$ :

$$
\begin{aligned}
& \text { Left Child }=2 k, \\
& \text { Right Child }=2 k+1, \\
& \text { Parent }=k / 2 \text { (integer division). }
\end{aligned}
$$

The advantage to using this type of sort is that every tree operation (insertion, removal, update) is of order $\log _{2} n$ where $\mathrm{n}$ is the number of elements in the tree, i.e., the number of close nodes at that step. Note that $n$ is of order $\sqrt{N}$ at every step, where $N$ is the total number of nodes in the mesh. By placing the close nodes into this structure, we keep the computational complexity on the order of $N \log _{2} N^{1 / 2}$.

\section{COMPUTATIONAL RESULTS}

We start by illustrating the efficiency of the proposed strategy in the presence of obstacles. The original method without extra boundary nodes and the proposed method are compared in Table I. The geometry is that of Figure 2 . The computational domain is the square $(0,6) \times(0,6)$. The source is located at $(0,0)$; a cylindrical obstacle of circular cross section is centered at $(3,3)$ and has radius 1 . In all the numerical experiments considered below, the value of the angle of repose $\delta$ is chosen as 40 degrees (for dry granular materials, values of $\delta$ between $30^{\circ}$ and $50^{\circ}$ are fairly typical).

As can clearly be seen in Table I, the scheme with the modified stencil retains first order convergence in the $L^{1}$ and $L^{2}$ norms even in the presence of the obstacle, unlike the algorithm 


\begin{tabular}{|l|l|l|l|l|l|l|l|l|l|l|l|l|}
\hline & \multicolumn{9}{|c|}{ standard stencil } & \multicolumn{9}{c|}{ modified stencil } \\
\hline $\mathrm{N}$ & $L^{1}$ & rate & $L^{2}$ & rate & $L^{\infty}$ & rate & $L^{1}$ & rate & $L^{2}$ & rate & $L^{\infty}$ & rate \\
\hline 25 & $2.52(0)$ & & $1.07(0)$ & & $8.99(-1)$ & & $1.17(0)$ & & $2.84(-1)$ & & $1.16(-1)$ & \\
50 & $1.45(0)$ & .80 & $5.94(-1)$ & .85 & $7.39(-1)$ & .28 & $2.83(-1)$ & 2.0 & $9.59(-2)$ & 1.6 & $4.54(-2)$ & 1.4 \\
100 & $6.36(-1)$ & 1.2 & $2.45(-1)$ & 1.3 & $3.10(-1)$ & 1.3 & $1.23(-1)$ & 1.2 & $4.26(-2)$ & 1.2 & $1.97(-2)$ & 1.2 \\
200 & $3.02(-1)$ & 1.1 & $1.20(-1)$ & 1.0 & $1.73(-1)$ & .84 & $3.29(-2)$ & 1.9 & $1.14(-2)$ & 1.9 & $1.22(-2)$ & .70 \\
400 & $1.52(-1)$ & .99 & $6.16(-2)$ & .97 & $8.49(-2)$ & 1.0 & $1.52(-2)$ & 1.1 & $5.32(-3)$ & 1.1 & $5.93(-3)$ & 1.0 \\
800 & $6.91(-2)$ & 1.1 & $2.91(-2)$ & 1.1 & $4.41(-2)$ & .95 & $2.06(-3)$ & 2.9 & $8.22(-4)$ & 2.7 & $3.11(-3)$ & .94 \\
1600 & $3.42(-2)$ & 1.0 & $1.45(-2)$ & 1.0 & $2.31(-2)$ & .93 & $4.82(-4)$ & 2.1 & $2.47(-4)$ & 1.7 & $1.68(-3)$ & .89 \\
3200 & $1.48(-2)$ & 1.2 & $6.36(-3)$ & 1.2 & $1.12(-2)$ & 1.0 & $1.15(-4)$ & 2.1 & $7.64(-5)$ & 1.7 & $8.83(-4)$ & .93 \\
\hline
\end{tabular}

Table II. Convergence study for formally second order methods in the presence of a cylindrical obstacle

with the standard stencil. First order convergence is not observed in the $L^{\infty}$ norm for either method because the solution is not smooth, see Figure 2.

Results related to formally second order methods are presented in Table II. The original method appears to convergence with order one in all three norms. The modified scheme is found to preserve second order accuracy in the $L^{1}$ norm even the presence of obstacles. The $L^{2}$ rates of convergence are slightly below 2 , while the method appears to converge with order 1 in the maximum norm. In all cases and all norms, the modified method shows much better accuracy than the standard method. It should be noted that second order convergence for the finer meshes was obtained only after rewriting the quadratic formula for solving (8) in a form that limits cancellation effects (standard IEC559 double precision was used throughout).

The last numerical experiment illustrates the feasibility of our method for problems with fully three dimensional geometrical properties, i.e., the non-cylindrical case. The configuration corresponding to Figure 3 is considered. The source is located on the inner wall of the top part of the (external) hopper. The dimensions of the external hopper are $11 \mathrm{ft}$ in height and $5 \mathrm{ft}$ in diameter; the obstacle is $7 \mathrm{ft}$ high.

\section{CONCLUSIONS}

In this paper, a model for the calculation of the volume and shape of large granular piles has been proposed. In case cylindrical obstacles and containers are exclusively considered, i.e., if the containment walls are all vertical, the problem admits an Eikonal formulation equivalent to that of classical travel time determination problems [17, 24]. For general obstacles and/or containers however, the analogy with travel time determination breaks down, as the "slowness" becomes a function of the unknown field.

The contributions of this paper are twofold. First, it is shown how to generalize marching methods such as the first and second order schemes proposed in [21] to problems involving obstacles (i.e., locally infinite slowness). The proposed method respects the marching character of the algorithm and thus is amenable to a Dijkstra like implementation of a heap sort algorithm [11], leading to a very fast method. Further, the algorithm does not lose accuracy in the neighborhood of the obstacle. Second, a new mathematical formulation of granular pile formation in complex geometries is proposed and successfully applied to the volume determination of bulk materials in bunkers. 


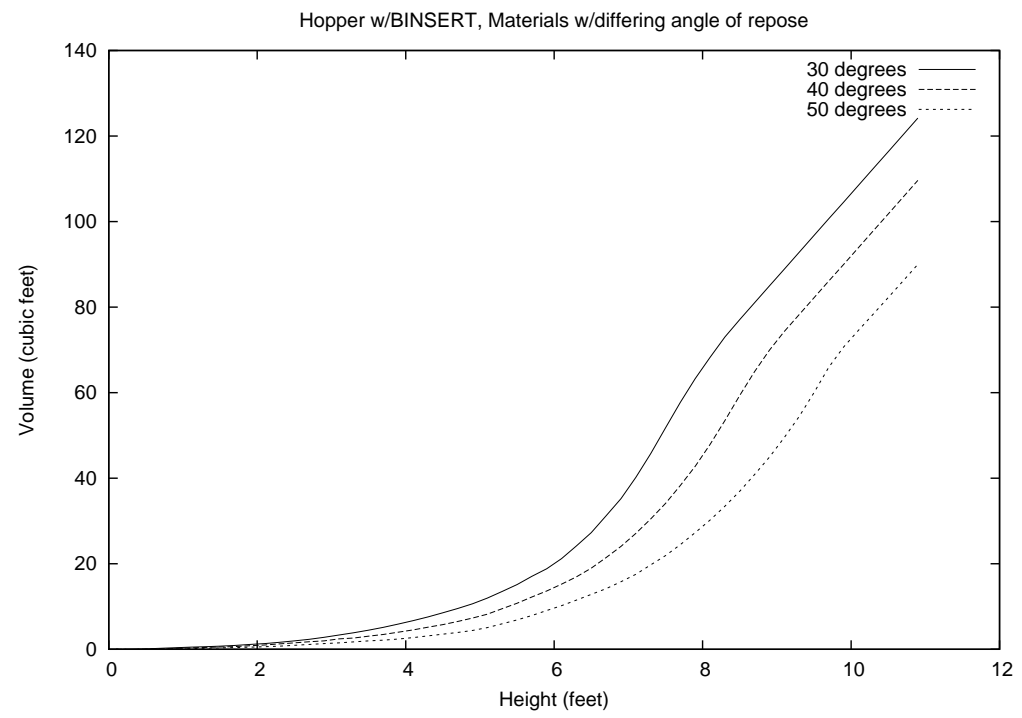

Figure 6. Volume vs height for the BINSERT geometry described in Figure 3.

\section{ACKNOWLEDGEMENTS}

The investigation of this problem was started by the authors, under the direction of Gremaud and Royal, during the Industrial Mathematics Modeling Workshop for Graduate Students held at North Carolina State University on July 22-July 30, 2002. The Workshop was supported by the National Science Foundation through grant DMS-0204515, with additional support provided by the Center for Research in Scientific Computation (CRSC), the Department of Mathematics at North Carolina State University and the Statistical and Applied Mathematical Sciences Institute (SAMSI). The support received from the National Science Foundation through grants DMS-9818900 and DMS-02044578 is also gratefully acknowledged.

\section{REFERENCES}

1. Aronsson, G. A mathematical model in sand mechanics: presentation and analysis, SIAM J. Appl. Math 1972; 22:437-458.

2. Aronsson, G., Evans, LC, Wu, Y. Fast/slow diffusion and growing sandpiles, J. Diff. Eq. 1996; 131:304335.

3. Bagnold, RA. The physics of blown sand and desert dunes, Metheun, 1941.

4. Behringer, RP. The dynamics of flowing sand, Nonlinear Sci. Today 1993; 3:1-15.

5. Bentley, J. Thanks, Heaps, Com. ACM 1985; 28(3):245-250.

6. Bouchaud, JP, Cates, ME, Ravi Prakash, J., Edwards, SF. Hysteresis and metastability in a continuum sandpile model, Phys. Rev. Lett. 1995; 74:1982-1985.

7. Boutreux, T., Raphaël, E., de Gennes, PG. Surface flows of granular materials: a modified picture for thick avalanches, Phys. Rev. E 1998; 58:4692-4700. 
8. Coulomb, CA. Essai sur une application des règles de maximis \& minimis à quelques problèmes de statique, relatifs à l'architecture, Mémoires de Mathématique 63 de Physique, présentés à l'Académie Royale des Sciences par divers Savans, $\mathcal{G}$ lûs dans ses Assemblées 1773; 7:343-382.

9. Crandall, MG., Lions, P.-L. Viscosity solutions of Hamilton-Jacobi equations, Trans. Amer. Math. Soc. 1983; 277:1-43.

10. Crandall, MG., Evans, LC., Lions, P.-L. Some properties of viscosity solutions of Hamilton-Jacobi equations, Trans. Amer. Math. Soc. 1984; 282:487-502.

11. Dijsktra, EW. A note on two problems in connexion with graphs, Numer. Math. 1959; 1:269-271.

12. Grasselli, Y., Herrmann, HJ. Shapes of heaps and in silos, Eur. Phys. J.B 1999; 10:673-679.

13. Grasselli, Y., Herrmann, HJ. Crater formation on a three dimensional granular heap, Granular Matter $2001 ; 3: 201-204$

14. Heyman, J. Coulomb's memoir on statics, Cambridge University Press, 1972.

15. Kapoor, S., Maheshwari, SN., Mitchell, JSB. An efficient algorithm for euclidean shortest paths among polygonal obstacles in the plane, Discrete Comput. Geom. 1997; 18:377-383.

16. Prigozhin, L., Zaltzman, B. Two continuous models for the dynamics of sandpile surfaces, Phys. Rev. E 2001; 63:041505-1-6.

17. Qian, J., Symes, WW. An adaptive finite difference method for traveltimes and amplitudes, Geophysics $2002 ; 67: 167-176$.

18. Schaeffer, DG. Instability in the evolution equations describing incompressible granular flow, J. Diff. Eq. $1987 ; 66: 19-50$.

19. Sethian, JA. Fast marching methods, SIAM Review 1999; 41:199-235.

20. Sethian, JA, Popovici, AM. 3-D traveltime computation using the fast marching method, Geophysics 1999 64:516-523.

21. Sethian, JA, Vladimirsky, A. Fast methods for the Eikonal and related Hamilton-Jacobi equations on unstructured meshes, Proc. Natl. Acad. Sci. USA 2000; 97(11):5699-5703.

22. Sethian, JA, Vladimirsky, A. Ordered upwind methods for static Hamilton-Jacobi equations: theory and algorithms, SIAM J. Numer. Anal. 2003; 41(1):325-363.

23. Tsitsiklis, J. Efficient algorithms for globally optimal trajectories, IEEE Transactions on Automatic Control 1995, 40:1528-1538.

24. van Trier, J., Symes, W. Upwind finite difference of traveltimes, Geophysics 1991; 56:812-821.

25. Williams, JWJ. Heapsort, Com. ACM 1964; 7(6):347-348. 